\title{
Optimal Operation Strategy for Biohydrogen Production
}

\author{
Ehecatl Antonio del Rio-Chanona, ${ }^{\dagger}$ Pongsathorn Dechatiwongse ${ }^{\ddagger}{ }^{\circ}$ Dongda \\ Zhang, ${ }^{\dagger}$ Geoffrey C. Maitland ${ }^{\ddagger}$ Klaus Hellgardt, ${ }^{\ddagger}$ Harvey Arellano-Garcia, ${ }^{\top}$ and \\ Vassilios S. Vassiliadis*,† \\ E-mail: vsv20@cam.ac.uk
}

Phone: +44 (0) 1223 330142. Fax: +44 (0) 1223334796

\begin{abstract}
Hydrogen produced by microalgae is intensively researched as a potential alternative to conventional energy sources. Scaling-up of the process is still an open issue, and to this end accurate dynamic modeling is very important. A challenge in the development of these highly nonlinear dynamic models is the estimation of the associated kinetic parameters. This work presents the estimation of the parameters of a revised Droop model for biohydrogen production by Cyanothece sp. ATCC 51142 in batch and fed-batch reactors. The latter reactor type results in an optimal control problem in which the influent concentration of nitrate is optimized which has never been considered previously. The kinetic model developed is demonstrated to predict experimental data to a high degree of accuracy. A key contribution of this work is the prediction
\end{abstract}

\footnotetext{
${ }^{*}$ To whom correspondence should be addressed

$\dagger$ Department of Chemical Engineering and Biotechnology, University of Cambridge, Pembroke Street, Cambridge CB2 3RA, UK

${ }^{\ddagger}$ Department of Chemical Engineering, Imperial College London, South Kensington Campus, London SW7 2AZ, UK

${ }^{\top}$ School of Engineering, University of Bradford, Richmond Road, Bradford, Yorkshire BD7 1DP, UK
} 
that hydrogen productivity can achieve $3365 \mathrm{~mL} / \mathrm{L}$ through an optimally controlled fed-batch process, corresponding to an increase of $116 \%$ over other recently published strategies.

\section{Introduction}

Global warming has been directly linked to the release of carbon dioxide $\left(\mathrm{CO}_{2}\right)$ by burning carbon-based energy resources ${ }^{1}$. Aside from the environmental impact, it does not seem to be sensible to permanently rely on limited and non-renewable conventional fuels for energy supply ${ }^{2}$. To reduce the production of $\mathrm{CO}_{2}$ and fulfill the increasing demands on energy supply, seeking novel sustainable and environmentally friendly energy resources is actively researched internationally.

Currently, hydrogen $\left(\mathrm{H}_{2}\right)$ produced by microorganisms is considered one of the fuels of the future with great potential for sustainability and environmental friendliness ${ }^{3}$. Different microorganisms such as green algae, cyanobacteria and purple non-sulfur bacteria are known to generate hydrogen. Chlamydomonas reinhardtii, an outstanding representative of green algae, produces biohydrogen by photosynthesis and utilizes water as the hydrogen source ${ }^{4}$. Instead of releasing $\mathrm{CO}_{2}$, C. reinhardtii fixes $\mathrm{CO}_{2}$ for its growth, indicating that the hydrogen generated in this process could be carbon-neutral ${ }^{5}$. Cyanobacteria are capable of producing hydrogen via different metabolic pathways including both photosynthesis and nitrogen-fixing pathways ${ }^{6}$. In particular, Cyanothece sp. ATCC 51142 attains the highest hydrogen production rates compared to other natural species, and $\mathrm{CO}_{2}$ can also be chosen as the carbon source in its photo-autotrophic growth period ${ }^{7}$. Purple non-sulfur bacteria have the advantage of continuously generating hydrogen after deprivation of a nitrogen source ${ }^{8}$. Additionally, their anoxygenic photosynthesis pathway significantly facilitates the commercialization of biohydrogen production, as hydrogen is only generated in anaerobic conditions regardless of the species of microorganisms ${ }^{4,6,9}$.

Extensive studies have been conducted to improve the productivity of hydrogen by dif- 
ferent microorganisms. Suppression of methanogenic activity for hydrogen production using mixed bacterial cultures has been investigated ${ }^{10}$. Effects of light intensity, temperature and nutrient ratio on microorganism growth and hydrogen production have also been examined $^{7,11-14}$. Different processes have also been designed to extend hydrogen production periods and improve biomass concentration ${ }^{15,16}$. Despite these efforts, there are unresolved problems that still seriously prevent industrialization of biohydrogen production processes. For example, in laboratory scale research, recent studies have concluded that light attenuation in photobioreactors (PBRs) can strongly limit algal and cyanobacterial growth and hydrogen production ${ }^{17,18}$. The configuration of a PBR is also found to affect the uniformity of culture mixing, light transmission and cell growth rate ${ }^{19,20}$. Furthermore, finding the optimal operating conditions such as incident light intensity, temperature and nutrient ratio is very demanding if it is carried out purely by experiments. On the other hand, recent research demonstrated that different reactor operation modes such as well-mixed mode and non-mixed mode can largely influence the productivity of biohydrogen production process ${ }^{21}$. As a result, it is essential to choose the suitable reactor type and to find the optimal operating conditions when scaling-up biohydrogen production processes.

Developing dynamic models to simulate and optimize fermentation processes have been widely accepted as the most effective method to solve the previously mentioned problems. A variety of dynamic models including the Monod model and the Droop model have been developed and modified by previous research for biohydrogen production ${ }^{22}{ }^{-24}$. With accurate and reliable dynamic models available, different reactor types and operation can be simulated, and the optimization of operating conditions such as nutrient ratio and dilution rate can be explored so as to reveal their maximal productive capability.

The use of dynamic models requires the use of state-of-the-art techniques in simulation and optimization so as to select the optimal reactor type and operation. The aim of the present study is twofold: to develop an accurate biohydrogen production model via parameter estimation, and the dynamic optimization of hydrogen production process models. The 
species investigated in the current work is Cyanothece sp. ATCC 51142 because of its distinctively high hydrogen production rate $^{7}$.

The key motivation for this work is to present original contributions in the area of modeling and optimizing fermentation processes, with particular emphasis on biohydrogen production, as follows:

1. Complete dynamic model identification via the use of rigorous dynamic optimization procedures

2. Verification of the derived models through comparison with experimental data

3. Use of the derived dynamic process model to predict the optimal operation of the underlying process

4. Investigate the impact on productivity of treating some traditionally constant operating parameters as controls

\section{Process Modeling and Optimization}

\section{Model Development}

Various dynamic models have been developed to simulate different growth phases of microorganisms ${ }^{?}$. Specific to the biohydrogen production process by green algae and cyanobacteria, five growth phases have been observed by recent experimental studies ${ }^{15,25}$ (i) the lag phase, (ii) the primary growth phase, (iii) the secondary growth phase, (iv) the stationary phase, and $(\mathrm{v})$ the decay phase ${ }^{21}$. In the primary growth phase, cyanobacteria grow rapidly because of the presence of essential nutrients, including nitrate (nitrogen source) and glycerol (carbon source). Once nitrate in the culture is consumed, the cyanobacterial growth phase shifts to the secondary growth phase. In this phase, cells continue growing by consuming the intracellular nitrogen source accumulated in the primary growth phase, and the activity of 
nitrogenase is recovered because of the absence of nitrate. The nitrogen-fixing process is also stimulated due to the activation of nitrogenase, which initiates the generation of hydrogen. After the rapid consumption of the intracellular nitrogen source, a brief stationary phase is observed which is followed by the decay phase where hydrogen is mainly generated.

To construct an accurate dynamic model for cyanobacterial hydrogen production, all of the growth phases except the lag phase have to be considered. As most of the present dynamic models are designed to simulate a single specific growth phase of microorganisms, such as the stationary phase or the first growth phase ${ }^{14,22-24}$, modifications have to be introduced with the aim to produce a complete process model that is capable of predicting all phases seamlessly. By comparing the characteristics of different dynamic models, a revised Droop model constructed in previous research ${ }^{21}$ is selected as it is capable of simulating the entire set of growth phases of cyanobacteria, except the lag phase. The revised Droop model is presented in Equations (1a)-(1i). Further details of model selection and construction can be found $\mathrm{in}^{21}$. As hydrogen production rates were found to be proportional to biomass concentration in our experiment work $^{15}$, the yield ratio of hydrogen to biomass, $Y_{H / X}$, is determined as $2.34 \mathrm{~mL} \cdot \mathrm{g}^{-1}$ and does not need to be estimated. 


$$
\begin{aligned}
& \frac{d \mathrm{X}}{d t}=\mu_{\max } \mathrm{X}\left(1-\frac{k_{q}}{q}\right) \frac{\mathrm{C}}{K_{C}+\mathrm{C}}-\mu_{d} \mathrm{X}^{2} \\
& \frac{d \mathrm{C}}{d t}=-Y_{C / X} \mu_{\max } \mathrm{X}\left(1-\frac{k_{q}}{q}\right) \frac{\mathrm{C}}{K_{C}+\mathrm{C}}+F_{i n} \mathrm{C}_{\mathrm{Fed}} \\
& \frac{d \mathrm{~N}}{d t}=-Y_{N / X} \mu_{\max } \mathrm{X} \frac{\mathrm{N}}{K_{N}+\mathrm{N}}+F_{i n} \mathrm{~N}_{F e d}(t) \\
& \frac{d \mathrm{q}}{d t}=Y_{q / X} \mu_{\max } \frac{\mathrm{N}}{K_{N}+\mathrm{N}}-\mu_{\max }\left(1-\frac{k_{q}}{q}\right) q \frac{\mathrm{C}}{K_{C}+\mathrm{C}} \\
& \frac{d \mathrm{O}}{d t}=Y_{O / X} \frac{\mathrm{N}}{K_{N}+\mathrm{N}} \mathrm{X}-Y_{d} \mathrm{X}^{2} f(\mathrm{O})+F_{i n} \mathrm{O}_{\mathrm{Fed}} F \\
& \frac{d \mathrm{H}}{d t}=Y_{H / X} \mathrm{X}(1-f(\mathrm{O})) f(\mathrm{~N}) \\
& f(\mathrm{~N})=0.5 \frac{\left((\mathrm{N}-100)^{2}\right)^{0.5}-(\mathrm{N}-100)}{\left[(\mathrm{N}-100)^{2}+0.1\right]^{0.5}} \\
& f(\mathrm{O})=\frac{\mathrm{O}}{\left(\mathrm{O}^{2}+0.1\right)^{0.5}} \\
& F_{\text {in }}=\frac{0.1}{720-T}
\end{aligned}
$$

where $\mathrm{N}$ is nitrate concentration $\left(\mathrm{mg} \cdot \mathrm{L}^{-1}\right), q$ denotes the normalized intracellular nitrogen source concentration,$k_{q}$ represents the normalized minimum intracellular nitrogen source concentration, $\mathrm{C}$ is glycerol concentration $(\mathrm{mM}), \mathrm{O}$ is oxygen concentration (\% of oxygen saturation in the solution), $\mathrm{H}$ is hydrogen production $\left(\mathrm{mL} \cdot \mathrm{L}^{-1}\right), \mathrm{X}$ is biomass concentration $\left(\mathrm{g} \cdot \mathrm{L}^{-1}\right) \cdot f(\mathrm{~N})$, and $f(\mathrm{O})$ are the switch functions to stimulate the production of hydrogen. 
When the culture is anaerobic $(\mathrm{O}=0.0)$ and the nitrate concentration is lower than its threshold $\left(\mathrm{N}<100 \mathrm{mg} \cdot \mathrm{L}^{-1}\right)$, hydrogen is generated, $(f(\mathrm{~N})=1$ and $f(\mathrm{O})=0.0)$; otherwise hydrogen production is inhibited $(f(\mathrm{~N})=0.0$ or $f(\mathrm{O})=1), F_{\text {in }}$ is the influent flow rate $\left(\mathrm{L} \cdot \mathrm{hr}^{-1}\right), \mathrm{C}_{\mathrm{Fed}}$ denotes the glycerol influent concentration $\left(\mathrm{mmol} \cdot \mathrm{L}^{-1}\right), \mathrm{O}_{\mathrm{Fed}}$ is oxygen influent concentration (\%), $\mathrm{N}_{\mathrm{Fed}}(t)$ is nitrate influent concentration $\left(\mathrm{mg} \cdot \mathrm{L}^{-1}\right)$ considered to be a manipulated variable, and $T(\mathrm{hr})$ is the switch time after which the inlet starts being administered to the reactor.

The above model simulates a fixed-volume fed-batch process very similar with that of a variable-volume fed-batch process ${ }^{21}$, in the current research only the fixed-volume fed-batch process is selected as the representative case.

\section{Parameter Estimation}

It is clearly important to estimate accurately the parameters of any process model in order to use it for evaluation and design purposes. Different parameter estimation techniques can be used in different scenarios. For problems without gross errors, weighted nonlinear leastsquares method is the best way to estimate the values of process variables. However, when a significant amount of noise exists either in the bio-system behavior or in the measurement of the states, least squares might not be the best method as outliers have a strong impact on the final fit. In an errors-in-variables-measured formulation (EVM) both errors in the input and output variables are taken into account. A major difficulty in solving this problem is that, since the error is accounted for in all the variables, the optimization is performed on both the parameters and the inputs, thus leading to problems with many degrees of freedom ${ }^{26}$. Mestimators are robust estimators, which can reduce the effect of large outliers or nullify their effect $^{27}$. Another type of robust estimator to outliers can be obtained by formulating the objective as an $\ell_{1}$-norm. Moreover, recent work has proposed an $\ell_{1}$-norm with a dead-band which enhances noise rejection and minimizes unnecessary parameter adjustment ${ }^{28}$.

In this work parameters are estimated through nonlinear least-squares, though there 
are other advanced regression formulations like those mentioned above the former has been chosen due to the measuring accuracy of experimental instruments. Parameter estimation for large nonlinear models has also been explored in ${ }^{26,29}$. In this section a description of the parameter estimation procedure followed in this work is presented

\section{Optimization problem formulation for parameter estimation}

Biohydrogen production in a fixed-volume fed-batch reactor is modeled by Equations (1a)(1i), where the set of parameters $p \in R^{9}$ is defined by:

$$
p=\left(p_{1}, p_{2}, p_{3}, p_{4}, p_{5}, p_{6}, p_{7}, p_{8}, p_{9}\right)=\left(\mu_{\max }, k_{q}, K_{C}, \mu_{d}, Y_{C / X}, Y_{N / X}, Y_{q / X}, Y_{O / X}, Y_{d}\right)
$$

To estimate the parameters in Equation (2) the following nonlinear least-squares optimization problem is formulated.

$$
\min _{p} \operatorname{LSQR}(p)=\sum_{i=1}^{N}\left(\hat{y}_{i}-y\left(t_{i}, p\right)\right)^{T} \Lambda_{i}\left(\hat{y}_{i}-y\left(t_{i}, p\right)\right)
$$

subject to:

$$
\begin{aligned}
& \frac{d x}{d t}=f(x(t), p) \\
& x_{l b} \leq x \leq x_{u b} \\
& p_{l b} \leq p \leq p_{u b} \\
& t=t_{0}, x=x_{0}
\end{aligned}
$$

where $x_{l b}, x_{u b}$ are lower and upper bounds, respectively, for the state variables, and $p_{u b}, p_{l b}$ 
are lower and upper bounds for the parameters, respectively. The output variables once the DAE system is solved are labeled $y$, while the experimental data is labeled as $\hat{y}$. The objective function is a general weighted least-squares function, where $\Lambda$ is the weighting matrix. Given the nonlinearity and stiffness of the DAE model in Equations (1a)-(1i), stiff system integration methods are required, hence the orthogonal collocation method over finite elements in time using Radau roots is used throughout this paper ${ }^{30}$. This method has proved to be highly efficient for discretizing dynamic systems, and computational costs are reduced significantly compared to simpler discretization schemes such as the implicit Euler method $^{31,32}$ which has been widely used in recent publications for dynamic model parameter estimation $^{22-24}$. 


\section{Optimization procedure}

The optimization problem is transcribed into an NLP through orthogonal collocation ${ }^{33}$ which can then be adressed by NLP solvers.

The implementation in this work is programmed in a Python environment, Pyomo. Py$\mathrm{omo}^{34}$ is a tool package for modeling optimization applications in Python and serves as an interface for the optimization solver IPOPT $^{35}$ (used as a library in Pyomo). It is used in our work to discretize and optimize parameter estimation problems. IPOPT is a software package for large-scale nonlinear optimization. It is designed to find (local) solutions of constrained nonlinear optimization problems with continuous variables. This solver is extremely efficient in handling nonlinear problems, and has the option to approximate the Hessian of the Lagrangian of the problem by a quasi-Newton method. This makes it a very effective solver in addressing nonlinear parameter estimation problems with nonlinear constraints (even though a Gauss-Newton Hessian is never supplied).

The optimization method used in this work is a local optimization method, hence it can only guarantee local solutions. The starting points of the optimization define the outcome of the fit and due to their importance, the state variables are given initial points as linear approximations of the profiles described by the data points in the case of monotonic profiles. When experimental data (and theory) suggested non-monotonic profiles, piecewise linear approximations are used instead. To ensure sufficient exploration of the impact of optimization starting points, a multistart method is employed which is explained further in the computational results section.

\section{Optimization of Biohydrogen Production}

The greatest challenge in biohydrogen production is the efficiency of the process, hence optimization of its operation is very important. In this subsection we describe the modelbased procedure adopted to optimize biohydrogen production for both a batch and a fedbatch process. This optimization will simulate a 30 day period which enable us to make a 
standardized comparison with existing published works ${ }^{15,36}$.

\section{Batch optimization}

The Equations (1a)-(1i) represent a fixed-volume fed-batch process. If the inlet flow $F_{\text {in }}=0$, then we can describe a batch reactor model. With the former constraint once the parameters are estimated, we proceed to find the optimal operating conditions that maximize hydrogen yield for the batch process. There are two crucial operating conditions to optimize hydrogen production: the initial nitrate concentration and the number of runs in a 30 day period. Optimizing nitrate initial concentration is straightforward, however optimizing the number of runs for the batch process results in a mixed-integer nonlinear programming problem (MINLP). To solve this MINLP a branch and bound (B\&B) procedure is used ${ }^{37}$.

The relaxed optimization problem is discretized by orthogonal collocation over 100 elements in time, with 3 collocation points per element. The previous discretization results in an optimization problem with approximately 4800 variables and 7200 constraints, in total. Once the optimization problem has been adapted to the orthogonal collocation formulation the resulting NLP is defined as follows:

Objective function

$$
\max _{t, \mathrm{~N}\left(t_{0}\right)} \mathrm{H}\left(t_{f}\right) \frac{720}{t+n \cdot 48}
$$

subject to:

$\underline{\text { Process dynamics }}$

$$
\dot{x}_{i, j}=f\left(x_{i, j}, \dot{x}_{i, j}, p\right)
$$

$\underline{\text { Collocation constraints }}$

$$
x_{i, j}=x_{i-1, K}+h_{i} \sum_{l=1}^{K} \Phi_{l}\left(\tau_{j}\right) \dot{x}_{i, l}
$$


Continuity constraints

$$
x_{i, 0}=x_{i-1, K}
$$

$\underline{\text { Initial conditions }}$

$$
x_{1,0}\left(t_{0}\right)=x_{0}
$$

$\underline{\text { Integration horizon }}$

$$
0 \leq t \leq t_{f}
$$

Bounds

$$
20 \leq t_{f} \leq 720 \quad \mathrm{~N}\left(t_{0}\right)_{l b} \leq \mathrm{N}\left(t_{0}\right) \leq \mathrm{N}\left(t_{0}\right)_{u b}
$$

where $t_{f}$ refers to the final integration time for each run, such that $\frac{720}{t_{f}}=n$, where $n$ is the optimal number of runs, $\mathrm{N}\left(t_{0}\right)$ is the initial nitrate concentration, for a cleaning time of 48 hours between runs ${ }^{15}$, appearing in the objective function. Because the current research aims to discover the maximal capacity of cyanobacterial hydrogen production, the total amount of biomass will be fixed at $0.2 \mathrm{~g}^{3,15}$ in this optimization study, while the other design conditions can be found in Table 1. A multistart scheme was used to identify better solutions, using 50 different starting points. From the 50 starting points 28 converged to 2.45 process runs, 11 converged to process 2.98 runs, 7 converged to process 4.02 runs, and 4 did not converge. This highlights the fact that this problem is nonconvex and has many local solutions. The average computational time for each computational optimization execution was 55 seconds, with a total of 2,750 seconds for all 50 starting points on an Intel Core i5, 4 GB RAM 2.53 GHz computer. As mentioned before, once the optimal value for this relaxed MINLP was found, a branch and bound scheme was employed which identified the optimal operating 
conditions as reported in the results section. This problem has the number of runs as an integer valued variable and is discretized into 100 elements in time, with 3 collocation points per element.

Table 1: Operating conditions of batch process

\begin{tabular}{l|l}
\hline Initial biomass concentration & $0.2 \cdot \mathrm{n}^{-1}\left[\mathrm{~g} \cdot \mathrm{L}^{-1}\right]$ \\
Operating time & $\mathrm{t} \in[20,720][\mathrm{hr}]$ \\
Total operating time & $720[\mathrm{hr}]$ \\
Initial oxygen concentration & $20 \%$ \\
Initial nitrate concentration & $\mathrm{N}_{0} \in[1.0,3000]\left[\mathrm{mg} \cdot \mathrm{L}^{-1}\right]$ \\
Initial glycerol concentration & $50\left[\mathrm{mmol} \cdot \mathrm{L}^{-1}\right]$ \\
Initial nitrogen quota & 1.0 \\
Initial hydrogen production & $0.0[\mathrm{~mL}]$ \\
Number of runs & 2 \\
Cleaning time & $48[\mathrm{hr}]$ \\
\hline
\end{tabular}

\section{Fed-batch process optimization}

An optimal control problem was formulated to optimize hydrogen production in the fedbatch process. Two manipulated variables were taken into account, the switching time $T$ which determines the time when nitrate starts to be administered into the reactor, and the manipulated variable $\mathrm{N}_{F e d}(t)$ which is the nitrate concentration of the influent. This last variable is allowed to change value once per day in a 30 day experiment, but otherwise remains constant during each 24 hour period (piecewise-constant control function). Operating conditions can be found in Table 2. The biohydrogen production model yields the following optimal control problem for a fed-batch reactor

Objective function

$$
\max _{T, \mathrm{~N}_{F e d}(t)} \mathrm{H}\left(t_{f}\right)
$$

subject to:

Equations (1a)-(1i) 
When orthogonal collocation is applied to the problem discretized above, it results in an NLP of considerable size. Direct solution of the entire set of constraints and variables resulting from the orthogonal collocation discretization was found to be difficult to converge. For this reason the SALA augmented Lagrangian decomposition method was used ${ }^{38}$. Decomposition algorithms have been found to be useful to initialize a dynamic optimization problem when good initial estimates are hard to find ${ }^{39}$. According to this approach, the discretized problem was decomposed into three decoupled subproblems. Each subproblem was discretized by orthogonal collocation into 100 elements and 3 collocation points, for an overall problem of 300 elements. In terms of overall size, the complete discretized problem involves 28,800 constraints and 14,400 variables. The outer coordination algorithm took 203 iterations to converge, each iteration in average had a computational cost of 163 seconds, the overall computational time was 9 hrs 12 minutes on an Intel Core i5, 4 GB RAM $2.53 \mathrm{GHz}$ computer. With good initialization points from the above framework, the non-decomposed problem presented a computational time of 152 seconds.

Table 2: Operating conditions of fed-batch process

\begin{tabular}{l|l}
\hline Initial biomass concentration & $0.2\left[\mathrm{~g} \cdot \mathrm{L}^{-1}\right]$ \\
Total operating time & $720[\mathrm{hr}]$ \\
Initial oxygen concentration & $20 \%$ \\
Initial nitrate concentration & $150\left[\mathrm{mg} \cdot \mathrm{L}^{-1}\right]$ \\
Initial glycerol concentration & $50\left[\mathrm{mmol} \cdot \mathrm{L}^{-1}\right]$ \\
Initial nitrogen quota & 1.0 \\
Initial hydrogen production & $0.0[\mathrm{~mL}]$ \\
Oxygen inlet concentration & $20 \%$ \\
Glycerol inlet concentration & $50\left[\mathrm{mmol} \cdot \mathrm{L}^{-1}\right]$ \\
\hline
\end{tabular}




\section{Results and discussion}

\section{Parameter estimation}

The resulting model from the parameter estimation least-squares optimization problem in Equations (3a)-(3e) is depicted in Figure (1), where the profiles for the experimental points, the initial guess, and the fitted model are shown.
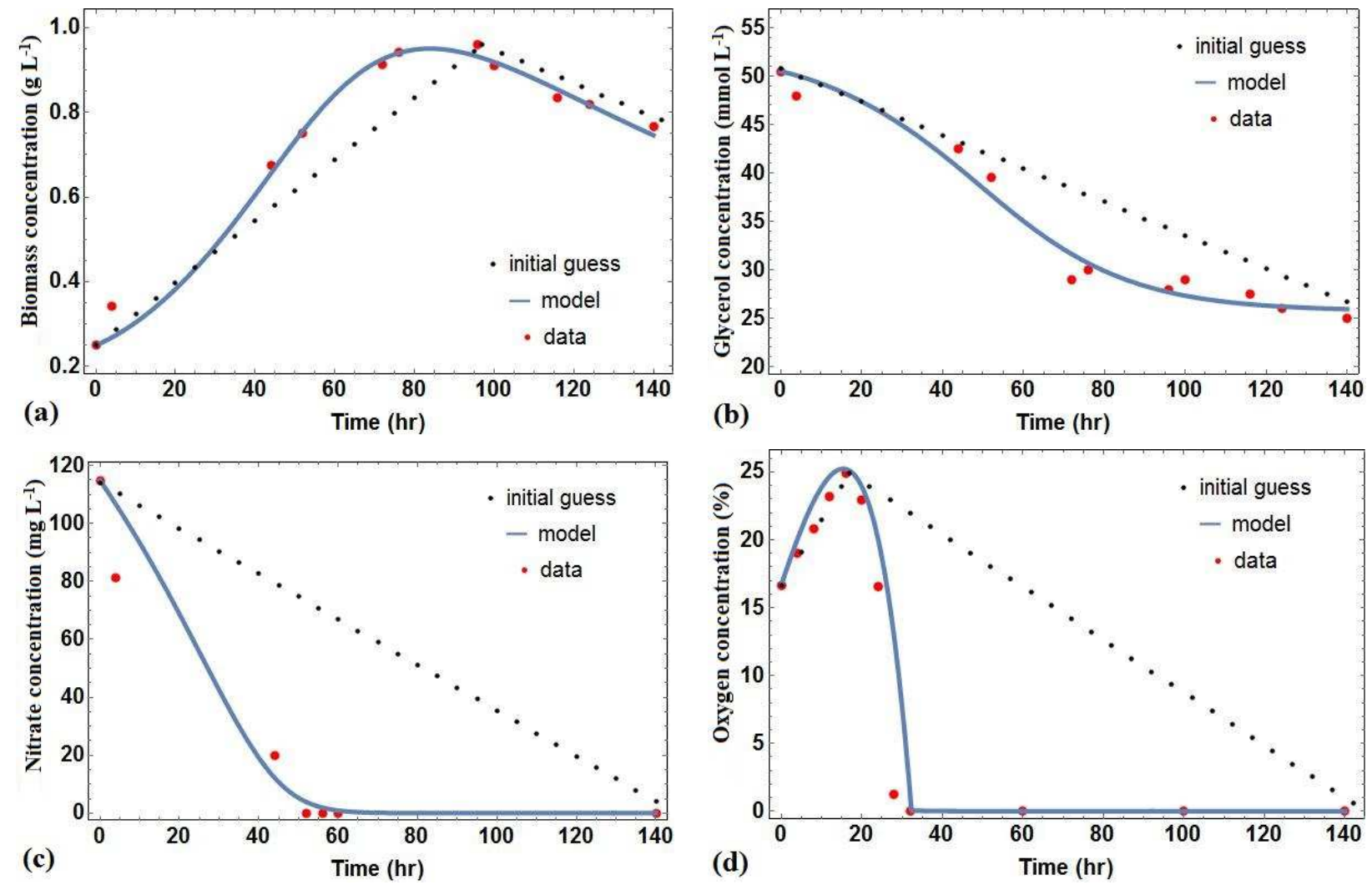

Figure 1: Comparison of simulation and experimental results. (a) biomass concentration. (b) glycerol concentration. (c) nitrate concentration. (d) oxygen concentration.

The parameter values solving the least-squares fitting optimization problem are presented in Table (3). In the current experiment, nitrate is the only limiting nutrient as the effect of glycerol on cyanobacterial growth and hydrogen production is negligible since it is always in excess. Therefore, $K_{C}$ is calculated as zero and the term $\frac{\mathrm{C}}{K_{C}+\mathrm{C}}$ accounting for the effect of glycerol becomes equal to 1.

Confidence intervals can be computed through the parameter estimation process. The 
covariance matrix for the estimated parameters can be approximated by the inverse of the reduced Hessian at the optimal solution. Confidence intervals can then be obtained from the trace of this approximated covariance matrix following standard procedures ${ }^{40}$. Due to the high nonlinearity and complexity of modeling metabolic kinetics, the assumption of computing the confidence intervals from the above framework may not hold. For this reason the confidence intervals presented in Table 3 must be understood as theoretical values. The detailed description of the model used in this work is presented $\mathrm{in}^{21}$. It is found that the simulation results are in good agreement with the experimental data as well as able to predict new processes. Therefore, the current model is suitable for further process design.

Table 3: Model identification parameter values

\begin{tabular}{ll|ll}
\hline Parameter & Value & Parameter & Value \\
\hline$\mu_{\max }\left[\mathrm{hr}^{-1}\right]$ & $0.04765 \pm 0.00822$ & $Y_{N / X}\left[\mathrm{mg} \cdot \mathrm{g}^{-1}\right]$ & $244.6 \pm 0.0635$ \\
$k_{q}$ & $0.6281 \pm 0.1727$ & $Y_{q / X}$ & $1.723 \pm 0.0011$ \\
$K_{C}\left[\mathrm{mmol} \cdot \mathrm{L}^{-1}\right]$ & $0.0 \pm 0.1223$ & $Y_{O / X}\left[\mathrm{~L} \cdot \mathrm{g}^{-1} \cdot \mathrm{hr}^{-1}\right]$ & $14.60 \pm 1.002$ \\
$\mu_{d}\left[\mathrm{~L} \cdot \mathrm{g}^{-1} \cdot \mathrm{hr}^{-1}\right]$ & $0.008559 \pm 0.000287$ & $Y_{d}\left[\mathrm{~L} \cdot \mathrm{g}^{-1}\right]$ & $26.22 \pm 0.8309$ \\
$K_{N}\left[\mathrm{mg} \cdot \mathrm{L}^{-1}\right]$ & $50.0 \pm 4.43$ & $Y_{C / X}\left[\mathrm{mmol} \cdot \mathrm{g}^{-1}\right]$ & $20.83 \pm 0.2467$ \\
\hline
\end{tabular}

\section{Optimal Operating Conditions in Batch Mode}

High biomass concentration in the culture is essential to efficiently generate hydrogen in a batch process. During the hydrogen production period cyanobacteria commence decaying due to the lack of nitrate. After a certain period most cells in the culture are dead, and fresh culture has to be reloaded in order to improve process efficiency. Although frequently renewing the culture in a photobioreactor can enhance hydrogen productivity, the increasing total cleaning time will reduce the hydrogen production period. It is thus essential that the operating time of each run to be optimized.

An optimal initial nitrate concentration exists due to the trade-off effects of nitrate concentration affecting hydrogen production. On the positive side, a higher nitrate concentration can enhance the growth of cyanobacteria, as hydrogen production rate is almost proportional to biomass concentration in a nitrate-limiting culture. On the negative side, the activity of 
nitrogenase will be inhibited if nitrate concentration is higher than a threshold $\left(100 \mathrm{mg} \cdot \mathrm{L}^{-1}\right.$ as observed in the current experiment), and hydrogen thereby will not be produced.

The resulting optimal batch operating conditions from the optimization problem defined in Equations $(4 \mathrm{a})-(4 \mathrm{~g})$ are: two runs $(n=2)$, an initial nitrate concentration $\mathrm{N}\left(t_{0}\right)=$ $2283.6 \mathrm{mg} \cdot \mathrm{L}^{-1}$, and a batch process duration $t_{f}=336 \mathrm{hr}$. The optimized batch process operation concentration profiles for a single run are shown in Figure (2).
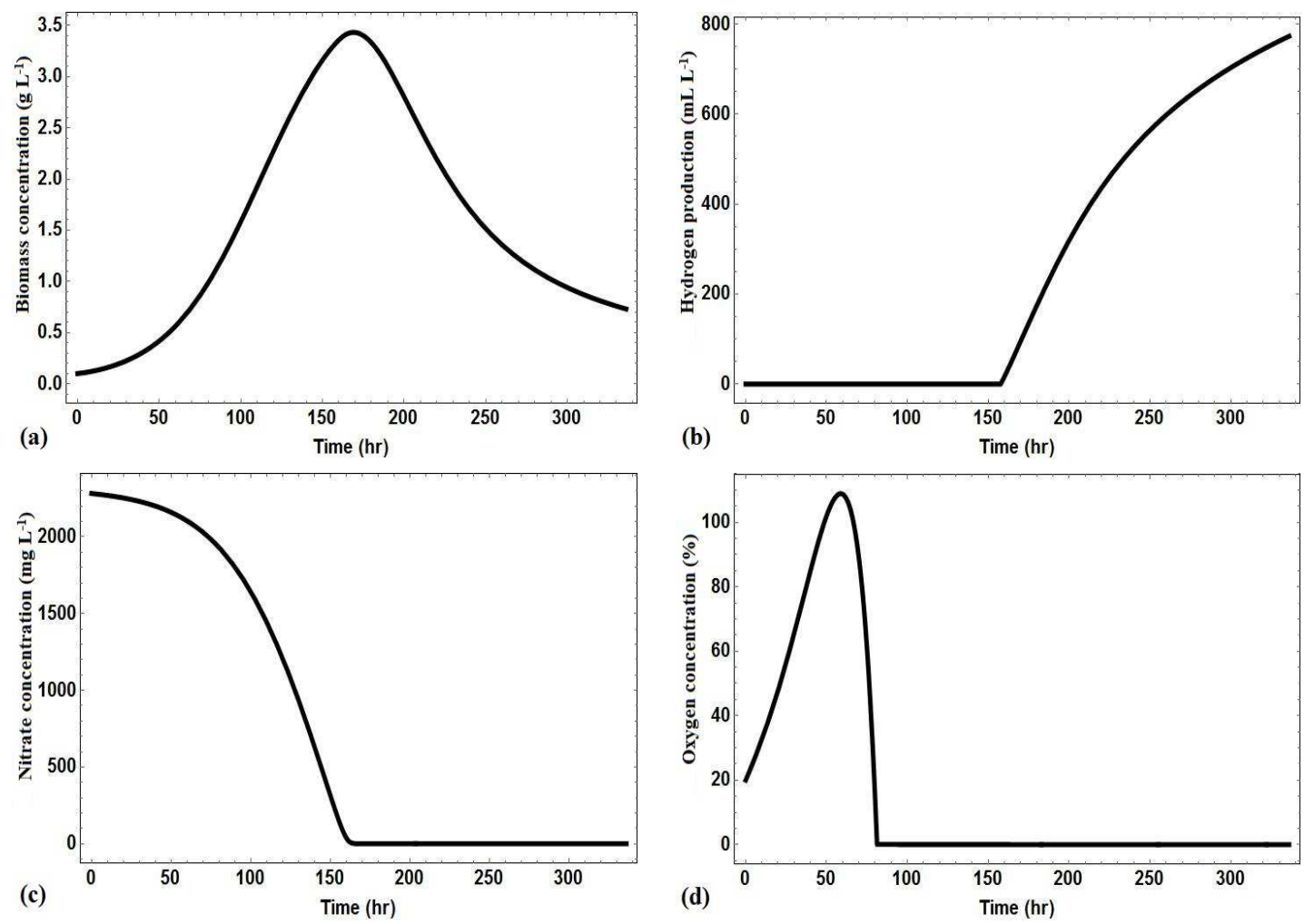

Figure 2: Optimized profiles of the batch process in each run. (a) biomass concentration. (b) hydrogen production. (c) nitrate concentration. (d) oxygen concentration.

During each run of the operation when the initial nitrate concentration is low, nitrate concentration in the culture rapidly drops below the threshold and because of this the hydrogen production period is extended (Figure 3(c)). However, total hydrogen production is severely limited due to the low biomass concentration caused by the low initial nitrate concentration. On the contrary, even if higher biomass concentration is obtained in the culture when the initial nitrate concentration is higher than its optimum, total hydrogen yield 
is still low because of the severely reduced hydrogen production period. This is because cyanobacteria have to spend more time in consuming nitrate to stimulate the activation of nitrogenase for hydrogen production (Figure 3(d)).
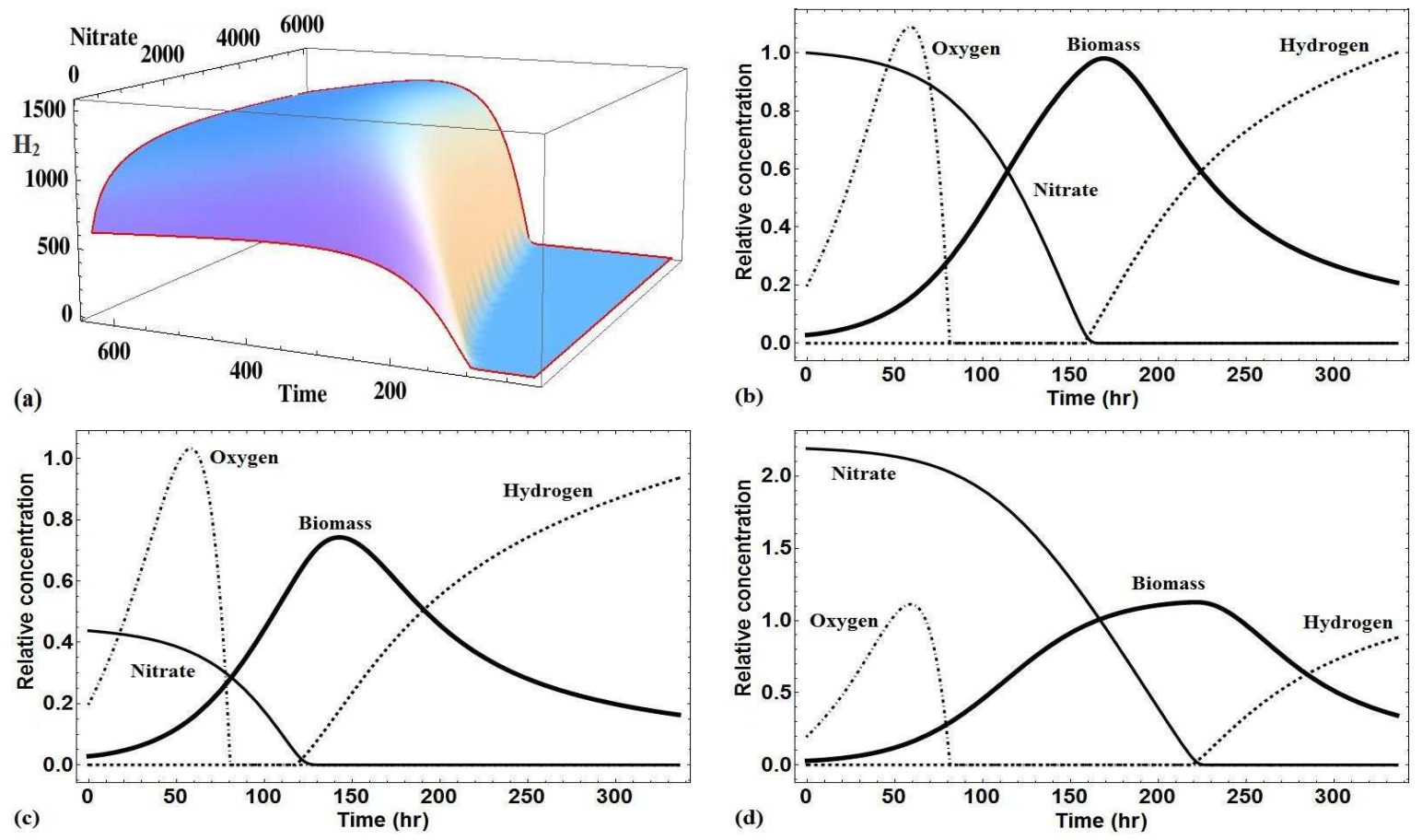

Figure 3: Biomass concentration $\left(\times 3.5 \mathrm{~g} \cdot \mathrm{L}^{-1}\right)$, nitrate concentration $\left(\times 2283 \mathrm{mg} \cdot \mathrm{L}^{-1}\right)$, oxygen concentration $(\times 100 \%)$ and hydrogen production $\left(\times 773 \mathrm{~mL} \cdot \mathrm{L}^{-1}\right)$ during the time course of batch processes with different initial nitrate concentrations. (a) hydrogen production with respect to the change of operating time for each run [hr] and initial nitrate concentration; (b) initial nitrate concentration is $2283 \mathrm{mg} \cdot \mathrm{L}^{-1}$; (c) initial nitrate concentration is $1000 \mathrm{mg} \cdot \mathrm{L}^{-1}$; (d) initial nitrate concentration is $5000 \mathrm{mg} \cdot \mathrm{L}^{-1}$.

The effects of operating time of each run on hydrogen production are very similar with those of the initial nitrate concentration. A longer operating time at each run may lead to a longer hydrogen production period, but the increased decay of cells is found to offset the advantage of a longer hydrogen production period. On the other hand, a shorter operating time for each run requires the initial biomass concentration to be very dilute. Hence, a longer cell growth period is necessary to increase biomass concentration, which decreases the hydrogen production period in each operation. The total hydrogen production period thereby is also reduced. 
The high nonlinearity of the batch process highlights the importance of robust and efficient modeling procedures. Given the discussion above, it becomes clear that the methodology followed throughout this contribution is necessary to describe accurately the process dynamics. On the other hand, the procedure followed to optimize the resulting MINLP to find optimal operating conditions guarantees a high quality solution, though practically difficult to certify the global optimality of the solutions obtained with dynamics such as the one describing the current system. It should be noted that the resulting optimization models are described by nonlinear equality constraints, which are many in numbers as is the case of the variables involved. The model is demonstrated numerically to be nonconvex, via the multistart policy, exhibiting many local optimal solutions.

\section{Optimal Operating Control Policy for the Fed-batch Process}

The resulting optimal switching time for the fed-batch process was $T=42 \mathrm{hr}$, and the optimal profiles for the dynamic optimization of the model in Equations (5) and Equations (1a)-(1i) are shown in Figure (4). 

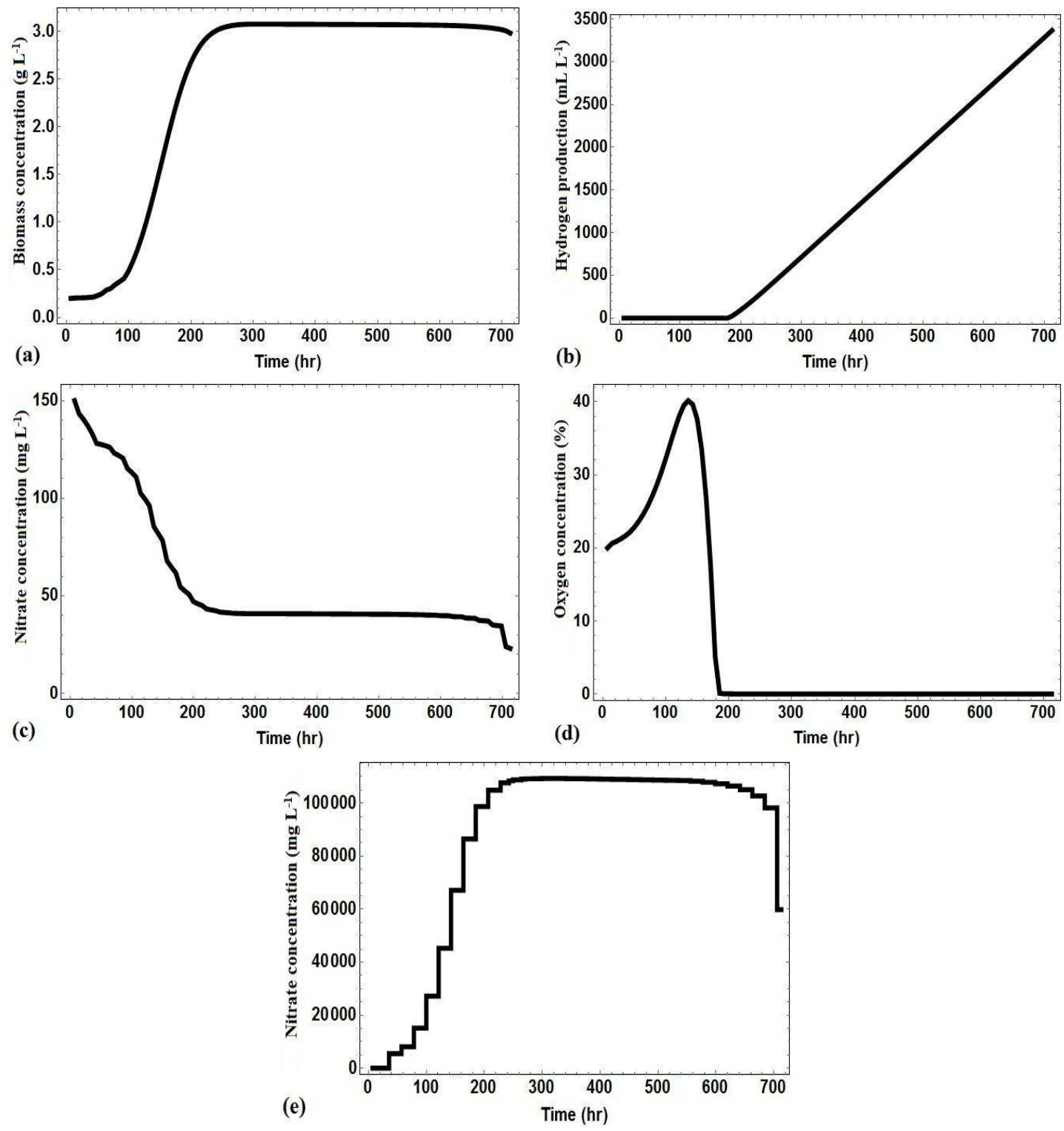

Figure 4: Optimized profiles of key state variables of the fed-batch process. (a) biomass concentration. (b) hydrogen production. (c) nitrate concentration in the culture. (d) oxygen concentration. (e) influent nitrate concentration (manipulated variable of the system).

The fed-batch process reaches a productivity of $3365 \mathrm{~mL} / \mathrm{L}$, this result constitute a significant improvement compared to both the experimental based optimized multiple batch process $(864 \mathrm{~mL} / \mathrm{L})^{41}$, and modelling-based optimized fed-batch process $(1560 \mathrm{~mL} / \mathrm{L})^{21}$. The optimal switching time is found to be at the $42^{\text {nd }}$ hour, where the initial nitrate in the culture is almost completely consumed. Nitrate concentration in the influent increases with operating time until cyanobacteria reach the stationary phase. Following this, the inlet 
nitrate concentration remains constant until the end of the experiment.

The increasing concentration of nitrate influent maintains a high cyanobacterial growth rate, and at the same time a rapid nitrate uptake rate in the culture. Given these conditions, nitrate concentration in the culture rapidly drops below its threshold to facilitate hydrogen production.

At the beginning of the feeding phase, the consumption rate of nitrate in the culture is slow due to the low biomass concentration. An increase of nitrate concentration in the culture is observed when the influent nitrate concentration is very high, as the nitrate addition rate is higher than the consumption rate (Figure 5(b)). This increase delays the start of hydrogen production because nitrogenase is only active at low nitrate concentration conditions (Figure 5(b)). If the accumulation of nitrate exceeds the maximum consumption capacity of the culture and nitrate is continuously added, the culture will become nitrate excessive and hydrogen is not possible to be generated during the entire processing period.
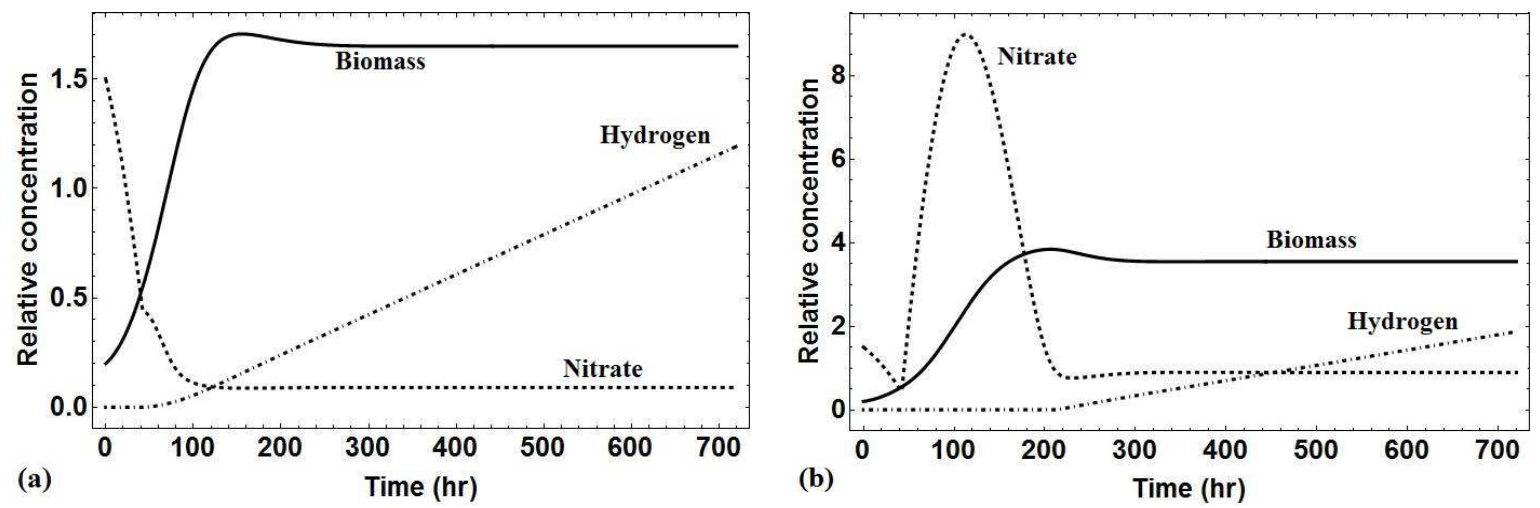

Figure 5: Biomass concentration $\left(\times 1.0 \mathrm{~g} \cdot \mathrm{L}^{-1}\right)$, nitrate concentration $\left(\times 100 \mathrm{mg} \cdot \mathrm{L}^{-1}\right)$ and hydrogen production $\left(\times 2000 \mathrm{~mL} \cdot \mathrm{L}^{-1}\right)$ in fed-batch process at different operating conditions. (a) the influent nitrate concentration is $2 \times 10^{4} \mathrm{mg} \cdot \mathrm{L}^{-1}$; (b) the influent nitrate concentration is $1.8 \times 10^{5} \mathrm{mg} \cdot \mathrm{L}^{-1}$.

However, if the influent nitrate concentration is always kept at a low concentration, cyanobacteria cannot fully grow because of the lack of nitrate (Figure 5(a)). Although nitrogenase activity can be recovered earlier, total hydrogen yield in this case is greatly restricted by the low biomass concentration (Figure 5(a)). Furthermore, the lack of nitrate in the cul- 
ture can lead to a strong anaerobic respiration of cyanobacteria, and acidic products excreted by the anaerobic respiration such as acetate will increase the cell death rate. The reason why the optimal switching time is determined at the $42^{\text {nd }}$ hour is that nitrate concentration in the culture is still high enough to maintain the rapid growth of cyanobacteria before this time, and an extra addition of nitrate from the influent will result in the accumulation of nitrate. If the switching time is much later than the optimal value, the decay of cyanobacteria will be obvious and biomass concentration will drop significantly, therefore hydrogen production will also be reduced.

Designing a viable fed-batch process for the bioproduction of hydrogen translates into higher efficiency productivity. The optimal control problem takes into account practical constraints in running such processes, such as allowing the inlet nitrate concentration to change once every 24 hours. Comparing the simulation results, the fed-batch process which allows inlet nitrate concentration to change, yields twice the amount of hydrogen as the standard multi-run optimized batch-process.

\section{Conclusions and future work}

This work proposes a systematic framework for proposing a model, verifying the model, and optimizing the productivity of the system. An original strategy to enhance productivity was developed, the hydrogen production yield predicted in this work is higher than any previously reported in the relevant literature, with $116 \%$ increase over the most recently published productivity ${ }^{21}$, and twice as high compared to that of a fully optimized multistage batch reactor.

To obtain these results, a traditionally static variable (inlet concentration of a nutrient for a fed-batch fermenter) is treated as a manipulated variable, and productivity is enhanced through dynamic optimization, constituting an original observation and contribution arising from this work. Additionally, a transferable model construction methodology for nonlinear 
biochemical dynamic processes is presented.

Future work will focus on experimental implementation of the optimal control fed-batch procedure proposed so as to verify and fine-tune the applicability of the proposed methodology. Also, model predictive control (MPC) of related bioprocesses will be explored, implementing online the dynamic optimization methodologies presented in this work. Finally, an economic analysis will be developed to assess feasibility of biohydrogen production by these new design strategies.

\section{Acknowledgments}

Author E. A. del Rio-Chanona would like to acknowledge CONACyT scholarship No. 522530 and the Secretariat of Public Education and the Mexican government for funding this project. Author P. Dechatiwongse is supported by a scholarship from the Royal Thai Government, Thailand. Solar Hydrogen Project was funded by the UK Engineering and Physical Sciences Research Council (EPSRC), project reference EP/F00270X/1. Author D. Zhang gratefully acknowledges the support from his family. The authors would also like to acknowledge Mr. Fabio Fiorelli for his meaningful suggestions. Finally, the authors wish to thank the anonymous referees for their insightful comments.

\section{Supporting Information Available}

This information is available free of charge via the Internet at http://pubs.acs.org/.

\section{Nomenclature}

$C_{F e d}$ glutamate inletconcentration

$F_{\text {in }}$ inlet flow

cee $H_{0}$ initial hydrogen production 
cee $H_{f}$ final hydrogen production

$K_{C}$ glutamate half velocity coefficient

$k_{q}$ minimum nitrogen quota

$c e e N_{0}$ initial nitrate concentration

$N_{F e d}$ nitrate inlet concentration

$c e e \mathrm{O}_{0}$ initial oxygen concentration

$O_{F e d}$ oxygen inlet concentration

$c e e q_{0}$ initial nitrogen quota

$T$ switch time

$c e e C_{0}$ initial glutamate concentration

cee $X_{0}$ initial biomass concentration

$Y_{C / X}$ glutamate yield coefficient

$Y_{d}$ oxygen consumption coefficient

$Y_{H / X}$ hydrogen yield coefficient

$Y_{N / X}$ nitrate yield coefficient

$Y_{O / X}$ oxygen yield coefficient

$Y_{q / X}$ nitrogen quota yield coefficient

$m u_{\max }$ maximum biomass specific growth rate

$m u_{d}$ biomass specific respiration rate

EVM errors in variables measured

MINLP mixed integer nonlinear programming

PBR photobioreactor 


\section{References}

(1) Kumar, K.; Dasgupta, C. N.; Nayak, B.; Lindblad, P.; Das, D. Development of suitable photobioreactors for CO2 sequestration addressing global warming using green algae and cyanobacteria. Bioresource Technology 2011, 102, 4945-4953.

(2) Brennan, L.; Owende, P. Biofuels from microalgae-A review of technologies for production, processing, and extractions of biofuels and co-products. Renewable and Sustainable Energy Reviews 2010, 14, 557-577.

(3) Elsharnouby, O.; Hafez, H.; Nakhla, G.; El Naggar, M. H. A critical literature review on biohydrogen production by pure cultures. International Journal of Hydrogen Energy 2013, 38, 4945-4966.

(4) Melis, a.; Zhang, L.; Forestier, M.; Ghirardi, M. L.; Seibert, M. Sustained photobiological hydrogen gas production upon reversible inactivation of oxygen evolution in the green alga Chlamydomonas reinhardtii. Plant physiology 2000, 122, 127-136.

(5) Antal, T. K.; Volgusheva, A. a.; Kukarskih, G. P.; Krendeleva, T. E.; Rubin, A. B. Relationships between H2 photoproduction and different electron transport pathways in sulfur-deprived Chlamydomonas reinhardtii. International Journal of Hydrogen Energy 2009, 34, 9087-9094.

(6) Min, H.; Sherman, L. a. Hydrogen production by the unicellular, diazotrophic cyanobacterium cyanothece sp. Strain ATCC 51142 under conditions of continuous light. Applied and Environmental Microbiology 2010, 76, 4293-4301.

(7) Bandyopadhyay, A.; Stöckel, J.; Min, H.; Sherman, L. a.; Pakrasi, H. B. High rates of photobiological H2 production by a cyanobacterium under aerobic conditions. Nature communications 2010, 1, 139. 
(8) McKinlay, J. B.; Oda, Y.; Ruhl, M.; Posto, A. L.; Sauer, U.; Harwood, C. S. Nongrowing rhodopseudomonas palustris increases the hydrogen gas yield from acetate by shifting from the glyoxylate shunt to the tricarboxylic acid cycle. Journal of Biological Chemistry 2014, 289, 1960-1970.

(9) Oh, Y. Photoproduction of hydrogen from acetate by a chemoheterotrophic bacterium $\backslash$ emph\{Rhodopseudomonas palustris\} P4. International Journal of Hydrogen Energy 2004, 29, 1115-1121.

(10) Hernández-Mendoza, C. E.; Buitrón, G. Suppression of methanogenic activity in anaerobic granular biomass for hydrogen production. Journal of Chemical Technology $\&$ Biotechnology 2014, 89, 143-149.

(11) Yang, H.; Zhang, J.; Wang, X.; Jiangtao, F.; Yan, W.; Liejin, G. A newly isolated Rhodobacter sphaeroides HY01 with high hydrogen production performance. International Journal of Hydrogen Energy 2014, 39, 10051-10060.

(12) Muñoz Páez, K. M.; Ruiz-Ordáz, N.; García-Mena, J.; Ponce-Noyola, M. T.; Ramos-Valdivia, A. C.; Robles-González, I. V.; Villa-Tanaca, L.; Barrera-Cortés, J.; Rinderknecht-Seijas, N.; Poggi-Varaldo, H. M. Comparison of biohydrogen production in fluidized bed bioreactors at room temperature and $35 \hat{\mathrm{A}}^{\circ} \mathrm{C}$. International Journal of Hydrogen Energy 2013, 38, 12570-12579.

(13) Escamilla-Alvarado, C.; Ríos-Leal, E.; Ponce-Noyola, M. T.; Poggi-Varaldo, H. M. Gas biofuels from solid substrate hydrogenogenic-methanogenic fermentation of the organic fraction of municipal solid waste. Process Biochemistry 2012, 47, 1572-1587.

(14) Alagesan, S.; Gaudana, S. B.; Krishnakumar, S.; Wangikar, P. P. Model based optimization of high cell density cultivation of nitrogen-fixing cyanobacteria. Bioresource Technology 2013, 148, 228-233. 
(15) Dechatiwongse, P.; Maitland, G.; Hellgardt, K. Demonstration of a two-stage aerobic/anaerobic chemostat for the enhanced production of hydrogen and biomass from unicellular nitrogen-fixing cyanobacterium. Algal Research 2015, 10, 189-201.

(16) Tamburic, B.; Dechatiwongse, P.; Zemichael, F. W.; Maitland, G. C.; Hellgardt, K. Process and reactor design for biophotolytic hydrogen production. Physical chemistry chemical physics : PCCP 2013, 15, 10783-94.

(17) Zhang, D.; Dechatiwongse, P.; del Rio-Chanona, E. A.; Hellgardt, K.; Vassiliadis, V. S. Modelling of Cyanobacteria Hydrogen Production Fermentation Process:Light Intensity and Temperature Effects on Cyanobacterial Growth and Hydrogen Production. Algal Research (In press) 2015,

(18) Schnurr, P. J.; Espie, G. S.; Allen, D. G. The effect of light direction and suspended cell concentrations on algal biofilm growth rates. 2014, 8553-8562.

(19) Zhang, D.; Dechatiwongse, P.; Hellgardt, K. Modelling light transmission, cyanobacterial growth kinetics and fluid dynamics in a laboratory scale multiphase photobioreactor for biological hydrogen production. Algal Research 2015, 8, 99-107.

(20) Tamburic, B.; Zemichael, F. W.; Crudge, P.; Maitland, G. C.; Hellgardt, K. Design of a novel flat-plate photobioreactor system for green algal hydrogen production. International Journal of Hydrogen Energy 2011, 36, 6578-6591.

(21) Zhang, D.; Dechatiwongse, P.; del Rio-Chanona, E. A.; Hellgardt, K.; Vassiliadis, V. S. Analysis of Cyanobacteria Hydrogen Photoproduction Process via Model Identification and Process Simulation. Chemical Engineering Science 2015, 128, 130-146.

(22) Xie, G.-J.; Liu, B.-F.; Xing, D.-F.; Nan, J.; Ding, J.; Ren, N.-Q. Photo-fermentative bacteria aggregation triggered by L-cysteine during hydrogen production. Biotechnology for biofuels 2013, 6, 64 . 
(23) Obeid, J.; Magnin, J. P.; Flaus, J. M.; Adrot, O.; Willison, J. C.; Zlatev, R. Modelling of hydrogen production in batch cultures of the photosynthetic bacterium Rhodobacter capsulatus. International Journal of Hydrogen Energy 2009, 34, 180-185.

(24) Wang, Y.-Z.; Liao, Q.; Zhu, X.; Li, J.; Lee, D.-J. Effect of culture conditions on the kinetics of hydrogen production by photosynthetic bacteria in batch culture. International Journal of Hydrogen Energy 2011, 36, 14004-14013.

(25) Tamburic, B. A Study of the Growth and Hydrogen Production of Chlamydomonas reinhardtii. Ph.D. thesis, Imperial College London, 2012.

(26) Zavala, V. M.; Biegler, L. T. Large-scale parameter estimation in low-density polyethylene tubular reactors. Industrial and Engineering Chemistry Research 2006, 45, 78677881.

(27) Arora, N.; Biegler, L. T. Redescending estimators for data reconciliation and parameter estimation. Computers and Chemical Engineering 2001, 25, 1585-1599.

(28) Hedengren, J. D.; Shishavan, R. A.; Powell, K. M.; Edgar, T. F. Nonlinear Modeling, Estimation and Predictive Control in APMonitor. Computers \& Chemical Engineering 2014

(29) Safdarnejad, S. M.; Hedengren, J. D.; Baxter, L. L. Plant-level dynamic optimization of Cryogenic Carbon Capture with conventional and renewable power sources. Applied Energy 2015, 149, 354-366.

(30) Kameswaran, S.; Biegler, L. T. Convergence rates for direct transcription of optimal control problems using collocation at Radau points. Computational Optimization and Applications 2007, 41, 81-126.

(31) Faber, R.; Li, P.; Wozny, G. Sequential Parameter Estimation for Large-Scale Sys- 
tems with Multiple Data Sets. 1. Computational Framework. Industrial $\&$ Engineering Chemistry Research 2003, 42, 5850-5860.

(32) Hong, W.; Wang, S.; Li, P.; Wozny, G.; Biegler, L. T. A quasi-sequential approach to large-scale dynamic optimization problems. AIChE Journal 2006, 52, 255-268.

(33) Biegler, L. T. An overview of simultaneous strategies for dynamic optimization. Chemical Engineering and Processing: Process Intensification 2007, 46, 1043-1053.

(34) Hart, W. E.; Laird, C.; Watson, J.-P.; Woodruff, D. L. Pyomo - Optimization Modeling in Python; Springer Optimization and Its Applications; Springer US: Boston, MA, 2012; Vol. 67; pp 13-28.

(35) Wächter, A.; Biegler, L. T. On the implementation of an interior-point filter line-search algorithm for large-scale nonlinear programming. Mathematical Programming 2005, $106,25-57$.

(36) Boran, E.; Özgür, E.; Van Der Burg, J.; Yücel, M.; Gündüz, U.; Eroglu, I. Biological hydrogen production by Rhodobacter capsulatus in solar tubular photo bioreactor. Journal of Cleaner Production 2010, 18.

(37) Lawler, E. L.; Wood, D. E. Branch-and-Bound Methods: A Survey. Operations Research 1966, 14, 699-719.

(38) Hamdi, A. Two-level primal-dual proximal decomposition technique to solve large scale optimization problems. Applied Mathematics and Computation 2005, 160, 921-938.

(39) Safdarnejad, S. M.; Hedengren, J. D.; Lewis, N. R.; Haseltine, E. Initialization Strategies for Optimization of Dynamic Systems. Computers \& Chemical Engineering 2015,

(40) Reinhardt, H. E. Nonlinear Parameter Estimation (Yonathan Bard). 1975. 
(41) Dechatiwongse, P.; Srisamai, S.; Maitland, G.; Hellgardt, K. Effects of light and temperature on the photoautotrophic growth and photoinhibition of nitrogen-fixing cyanobacterium Cyanothece sp. ATCC 51142. Algal Research 2014, 5, 103-111. 